\title{
ONOMÁVAIN
}

Revista semestral de lingüística, filología y traducción

\section{Diseño y evaluación de una aplicación tecnológica para la enseñanza del español como lengua extranjera ${ }^{1}$}

Design and Evaluation of a Technology Application for Teaching Spanish as a Foreign Language

\section{Anita Ferreira Cabrera \\ Universidad de Concepción \\ Chile}

\section{Ana Vine Jara \\ Universidad de Concepción \\ Chile}

\author{
Jessica Elejalde \\ Universidad de Concepción \\ Chile
}

Anita Ferreira: Programa de Español como Lengua Extranjera (ele.udec.cl), Facultad de Humanidades y Arte, Universidad de Concepción, Chile. I Correo electrónico: aferreir@udec.cl

Ana Vine: Programa de Español como Lengua Extranjera (ele.udec.cl), Facultad de Humanidades y Arte, Universidad de Concepción, Chile.

Jessica Elejalde: Programa de Español como Lengua Extranjera (ele.udec.cl), Facultad de Humanidades y Arte, Universidad de Concepción, Chile. 


\section{Resumen}

En el ámbito de la Lingüística Aplicada, la Enseñanza de Lenguas Asistida por el Computador (del inglés, Computer Assisted Language Learning, CALL) ha tenido un papel importante en el mejoramiento del aprendizaje de lenguas extranjeras. Una de las problemáticas centrales de esta interdisciplina es cómo integrar de manera efectiva el uso de la tecnología en una metodología de enseñanza de lenguas. Acorde con el modelo metodológico de CALL, en este trabajo se describe y explica el desarrollo, la implementación y la evaluación de una aplicación tecnológica para la enseñanza del español como lengua extranjera (ELE). La fundamentación teórica se sustenta en los planteamientos teóricos del enfoque por tareas y la hipótesis de la interacción. La evaluación de la aplicación se llevó a cabo a través de un estudio cuasiexperimental sin grupo control, en el cual participaron 21 estudiantes de nivel intermedio (BI) de ELE. Los resultados evidencian la efectividad de un modelo semipresencial, puesto que los estudiantes incrementaron de manera significativa su aprendizaje lingüístico y comunicativo al término del proceso de intervención lingüística.

Palabras clave: metodología CALL; español como lengua extranjera; adquisición de segundas lenguas; enfoque basado en tareas.

\section{Abstract}

In the field of Applied Linguistics, Computer Assisted Language Learning, CALL, has played an important role in improving learning of foreign languages. A central problem is how to effectively integrate the use of technology in language teaching methodology. In line with the methodological model of CALL, this paper describes and explains the development, implementation and evaluation of a technology application for teaching Spanish as a Foreign Language (ELE). The theoretical foundation is based on the theory of the task-based approach and the interaction hypothesis. The evaluation of the application was carried out through a quasi-experimental study without a control group, in which 21 students of ELE at intermediate level (B1) participated. The results show the effectiveness of this model, since students significantly increased their linguistic and communicative learning at the end of the process of language intervention.

Keywords: Call methodology; Spanish as a foreign language; second language acquisition; task based approach.

1 Este artículo se sustenta en los desarrollos investigativos del proyecto Fondecyt No.1110812 "Un sistema tutorial inteligente para la focalización en la forma en la enseñanza del español como lengua extranjera”. 


\section{Introducción}

En el ámbito de la enseñanza de lengua asistida por el computador (del inglés, Computer Assisted Language Learning, CALL), la proliferación de recursos tecnológicos ha modificado las modalidades de enseñanza de las lenguas. Hoy en día es posible plantearse la enseñanza en distintos escenarios, ya sea presencial, semipresencial o a distancia. En este contexto, los avances en el campo de la investigación permiten el tratamiento de modelos de enseñanza eclécticos que utilizan elementos de la enseñanza a distancia y presencial (Gruba y Hinkelman, 2012; Lamy y Hampel, 2007). La enseñanza a distancia ofrece una variedad de input auténtico al estudiante, sin embargo, otras habilidades, como las orales, no siempre se ven favorecidas por los recursos tecnológicos, por lo que la práctica presencial sigue siendo aún muy relevante. En este contexto, una modalidad semipresencial que combine ambas modalidades se constituye en una buena opción para la enseñanza de la lengua. La problemática en estudio surge a partir de la necesidad de mejorar las habilidades comunicativas de los estudiantes de español como lengua extranjera (ELE), mediante el uso de recursos tecnológicos que apoyen el desarrollo de las habilidades orales. En este sentido, consideramos que la articulación de la metodología de CALL en una modalidad de enseñanza semipresencial, fundamentada en la hipótesis de la interacción y el enfoque por tareas, propicia un ambiente favorable para que los aprendientes mejoren su competencia comunicativa en ELE.

El trabajo que se presenta tiene dos objetivos principales: (1) operacionalizar el modelo metodológico de CALL (Hubbard, 1996) en el contexto del diseño e implementación de una aplicación tecnológica para la enseñanza de ELE y (2) evaluar el impacto de uso de dicha aplicación en el aprendizaje de ELE.

El artículo se organiza en tres secciones: en la sección 2 se delimitan los fundamentos teóri- cos que sustentan el estudio, en la sección 3 se describe la metodología de investigación para el desarrollo, implementación y evaluación de una aplicación tecnológica para la enseñanza de ELE y en la sección 4 se aborda las conclusiones del estudio realizado.

\section{Fundamentos teóricos}

\subsection{Enseñanza de lenguas asistida por computador en una modalidad semipre- sencial}

El desarrollo de una aplicación tecnológica para la enseñanza de la lengua conlleva una serie de ventajas, por ejemplo: promueve un mejor proceso de enseñanza y aprendizaje, permite entregar input auténtico, gracias a los recursos y avances tecnológicos (páginas web, blogs, plataformas, dispositivos móviles), y facilita la adaptación de dichos recursos a las necesidades individuales de los aprendientes. Acorde con autores como Hubbard (2004), Levy y Stockwell (2006), Lamy y Hampel (2007) y Ferreira (2007), la interdisciplina CALL se ha constituido en un área desde la cual provienen los fundamentos y las proposiciones concretas sobre cómo integrar el computador en una metodología de enseñanza de lenguas. Por otra parte, dicha interdisciplina se "nutre del trabajo empírico y teórico de otras áreas" (Ferreira, 2007: 273), entre ellas: la Lingüística Aplicada, la Adquisición de Segundas Lenguas (ASL), la informática educativa y la inteligencia artificial en lo que compete a sistemas tutoriales inteligentes para lenguas extranjeras (del inglés, Intelligent Tutorial Systems for Foreign Language).

La investigación en este ámbito se interesa en diseñar, evaluar y analizar modelos de enseñanza y aprendizaje, los cuales permiten optimizar la calidad y cantidad del input y la práctica en la lengua. Para ello, indaga sobre los diferentes usos y maneras de incluir el computador, la tecnología y las herramientas disponibles en el proceso de enseñanza-aprendizaje de lenguas. 
Actualmente, los recursos disponibles en línea permiten a los profesores de lenguas implementar modelos metodológicos donde se combine modalidades presenciales y en línea (del inglés e-learning) para apoyar el proceso de aprendizaje. En este contexto interdisciplinario, se realizan esfuerzos por investigar e implementar metodologías de enseñanza de lenguas que posibiliten el aprendizaje no solo en ambientes presenciales o no presenciales (e-learning), sino también semipresenciales (del inglés b-learning).

Dicha implementación se logra, por ejemplo, a través del uso de plataformas para la enseñanza y aprendizaje a distancia. En el ámbito de la enseñanza de ELE, el Instituto Cervantes ha desarrollado y comercializado con éxito su plataforma Aula Virtual de Español (AVE) y hoy en día es una de las más conocidas para la enseñanza de ELE (Coto, 2014). Algunas de las claves del éxito de este entorno de enseñanza son la perspectiva metodológica adoptada, el desarrollo técnico, el uso de los materiales desarrollados para cursos presenciales y semipresenciales, entre otras.

También se cuenta con amplias experiencias en proyectos de telecolaboración, videoconferencias y videcomunicación en la enseñanza de ELE y de otras lenguas, como el francés. A través de estos estudios se ha logrado mejorar las competencias comunicativas e interculturales de los estudiantes (López y Ballez, 2014; Vine y Ferreira, 2012; Jauregi, 2011; Jauregi, Gómez y Canto, 2010; Juan Lázaro, 2009; Jauregi, Canto y Ros, 2006). Sin embargo, en el ámbito de la enseñanza del ELE faltan estudios empíricos que muestren la efectividad de estas modalidades en cuanto al mejoramiento de la competencia comunicativa de los estudiantes.

La modalidad a distancia (e-learning) se refiere a la enseñanza-aprendizaje de la lengua que se entrega de manera no presencial (en línea). Este tipo de aprendizaje aprovecha los distintos recursos didácticos que Internet ofrece para hacer de la enseñanza una instancia interactiva y fácil de seguir. En esta modalidad, el profesor actúa como un tutor online, porque resuelve dudas, corrige las tareas, propone actividades, etc. El aspecto diferenciador es que estas acciones las realiza usando Internet como herramienta de comunicación.

En cambio, la modalidad semipresencial (b-learning) se refiere a una metodología de enseñanza mixta, que combina tanto formación presencial como no presencial a través de la inclusión de recursos tecnológicos. Dicha inclusión de recursos en línea apoya al estudiante por medio de la entrega de un input de calidad y auténtico, logrando así mejorar su proceso de aprendizaje y desarrollar su propia autonomía (Egbert, 2005; Hubbard, 2004; Ferreira, García y Morales, 2014). En esta modalidad el profesor asume su papel tradicional, pero se apoya en la tecnología para llevar a cabo su labor, en dos formas: como tutor online (tutorías no presenciales) y como profesor tradicional (clases presenciales). Ferreira (2007) señala que la forma en que se combinen ambas estrategias dependerá de las metodologías de enseñanza subyacentes y de las necesidades específicas de los alumnos.

En este mismo contexto, De Basterrechea y Juan Lázaro (2010) señalan que el término que en la actualidad más se menciona es el de enseñanza semipresencial (o blended learning). Se trata de una apuesta interesante en el ámbito de la enseñanza de lenguas, ya que permite el desarrollo de actividades y estrategias de interacción, tanto oral como escrita.

Algunos estudios que han implementado el modelo b-learning como, por ejemplo, el de Bañados (2006) han evidenciado la efectividad de dicho modelo en la enseñanza-aprendizaje del inglés. Bañados (2006) trabajó con un grupo de 39 estudiantes de inglés como LE, quienes luego de un periodo de trabajo de 15 semanas (en que se consideró el uso de la plataforma y sesiones presenciales) incrementaron notablemente sus habilidades comunicativas en cuanto al uso de 
la lengua. Por otra parte, el trabajo de Shih (2010) implementó el modelo b-learning a través del uso de blogs para la enseñanza-aprendizaje de inglés con fines específicos, mostrando también la efectividad en el aprendizaje.

En el trabajo de Ferreira y otros (2014), a través de un estudio experimental se combina la enseñanza basada en tareas y los principios metodológicos de CALL para la modalidad blearning. Los resultados muestran que el grupo experimental, que trabajó con el modelo combinado, incrementó su aprendizaje en cuanto a las habilidades lingüísticas en estudio en comparación con el grupo de control que utilizó una metodología presencial. Igualmente, los resultados proporcionan directrices válidas para el desarrollo de materiales que utilicen recursos tecnológicos para el aprendizaje de lenguas extranjeras. Como se puede observar, hay un número creciente de investigaciones en enseñanza del inglés con la modalidad b-learning, sin embargo, en ELE, se requiere de mayor investigación que aporte en el ámbito de las nuevas propuestas metodológicas que incorporen la modalidad objeto de estudio en esta investigación.

\subsection{Modelo metodológico de CALL}

La metodología de CALL define un conjunto de principios bajo el contexto de la incorporación del computador al proceso de enseñanzaaprendizaje de lenguas extranjeras. Se pone énfasis en la consistencia que debe darse entre el modelo de CALL y las diferentes metodologías de enseñanza de lenguas, como también la coherencia entre el desarrollo, evaluación e implementación. Este modelo debe ser un método neutral y flexible, que describa la relación entre los estudiantes, los profesores y los computadores (Hubbard, 1996).

El modelo de CALL se compone de tres módulos: desarrollo, implementación y evaluación (Hubbard, 1996), los cuales se encuentran relacionados entre sí, como se muestra en la figura 1.

\section{FIGURA 1}

Modelo metodológico de CALL (Hubbard, 1996)

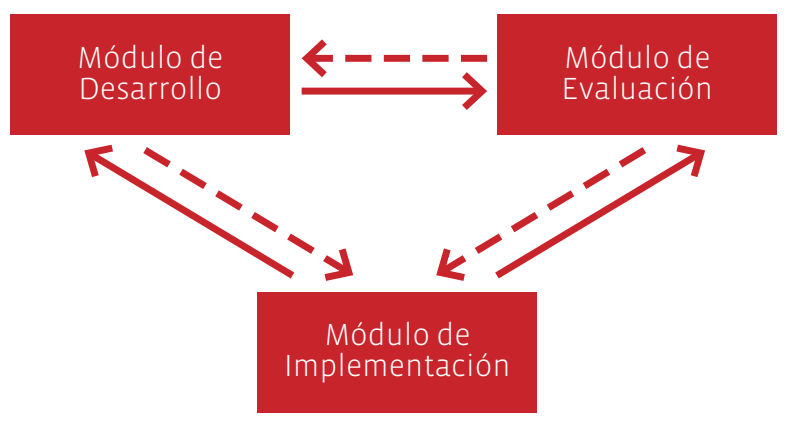

\subsubsection{Módulo de desarrollo}

Este módulo considera tres componentes: el enfoque de enseñanza, el diseño y los procedimientos. El enfoque considera dos aspectos relevantes, los supuestos lingüísticos y de aprendizaje, los que se combinan para definir el enfoque de enseñanza de la lengua que adoptará el desarrollador para una aplicación. Los supuestos lingüísticos proveen un conjunto de principios que se basan en la comprensión del desarrollador respecto a la naturaleza de la lengua que se enseña y la relativa importancia de los aspectos culturales y la estructura social. Los supuestos de aprendizaje proporcionan una serie de principios orientativos basados en la comprensión de la naturaleza del proceso de aprendizaje de una segunda lengua y en el rol que tiene el ambiente de aprendizaje en este proceso. En este componente se considera además el diseño de la interfaz de la aplicación tecnológica y los procedimientos para el uso de la aplicación con los alumnos. La interfaz debe representar cómo aparecerán las tareas en la pantalla, las opciones de control, las consideraciones de entrada (input), el feedback y las opciones de ayuda.

En el diseño, Hubbard (1996) menciona dos componentes esenciales: el perfil del estudiante y el programa de la aplicación. El primero se relaciona con los alumnos a quienes se dirige la aplicación, el nivel de proficiencia de los alumnos, la edad, la lengua materna, etc. El segundo está en 
concordancia con los objetivos lingüísticos del aprendizaje. A estos dos aspectos se agregan algunas consideraciones que implican la toma de decisiones, por ejemplo, respecto a las dificultades de la lengua que se está aprendiendo y de la aplicación tecnológica que se va a usar.

\subsubsection{Módulo de implementación}

Este módulo comprende una serie de elementos relacionados con el desarrollo de actividades mediante el uso de la tecnología, tales como: el acceso de los alumnos a los computadores, las actividades preparatorias antes de usar la aplicación, el monitoreo de los estudiantes mientras trabajan con la aplicación, el tiempo de estudio dedicado a cada sesión, los registros del estudiante en el programa, etc. Una vez que el estudiante ha finalizado la parte principal de la lección se deben tener en cuenta actividades de seguimiento con la finalidad de reforzar lo aprendido

\subsubsection{Módulo de evaluación}

La evaluación de una aplicación tecnológica para la enseñanza de lenguas se puede llevar a cabo desde dos perspectivas: 1) empírica a través de un estudio experimental o cuasi-experimental para medir el incremento en el aprendizaje de los alumnos en un contexto semipresencial y 2) el análisis valorativo de la aplicación computacional. Esta evaluación generalmente es realizada por expertos y alumnos del área por medio de un cuestionario de satisfacción de usuario.

Ferreira (2005) en su aplicación computacional "Si vas para Chile" realizó una evaluación de tipo valorativa, donde participaron un grupo de expertos y otro de estudiantes. La característica mejor evaluada de esta fue la calidad de la interfaz computacional en cuanto a los aspectos de la cultura y las costumbres chilenas.

\subsection{Hipótesis de la interacción}

En el paradigma cognitivo, el aprendizaje de idiomas se percibe como un proceso interno que ocurre en la mente del individuo y que contribuye al desarrollo de la lengua.

Aprender una segunda lengua implica no solo conocer aspectos fonéticos, gramaticales y léxicos de la lengua, sino también ponerlos en práctica, usarlos adecuadamente en contextos reales de comunicación. Por lo tanto, no solo son relevantes conceptos como input y output, sino también es importante el proceso de interacción que se da entre los aprendientes y otros hablantes (nativos o no nativos). Gass, Mackey y Pica (1998) y Gass, Behney y Plonsky (2013) afirman que el input es más eficaz cuando forma parte de la interacción con otros, y no se limita solo a un texto. Mediante el proceso de interacción se produce la negociación del significado y se logra un mayor entendimiento entre los estudiantes, por ejemplo, a través de las reformulaciones, simplificaciones, clarificaciones, etc. (Long, 1991), como se muestra en la figura 2, donde el output de un estudiante se convierte en input para su interlocutor a través de la interacción que se da entre ellos.

\section{FIGURA 2}

Modelo input-output en ASL (Adaptado de Lamy y Hampel, 2007)

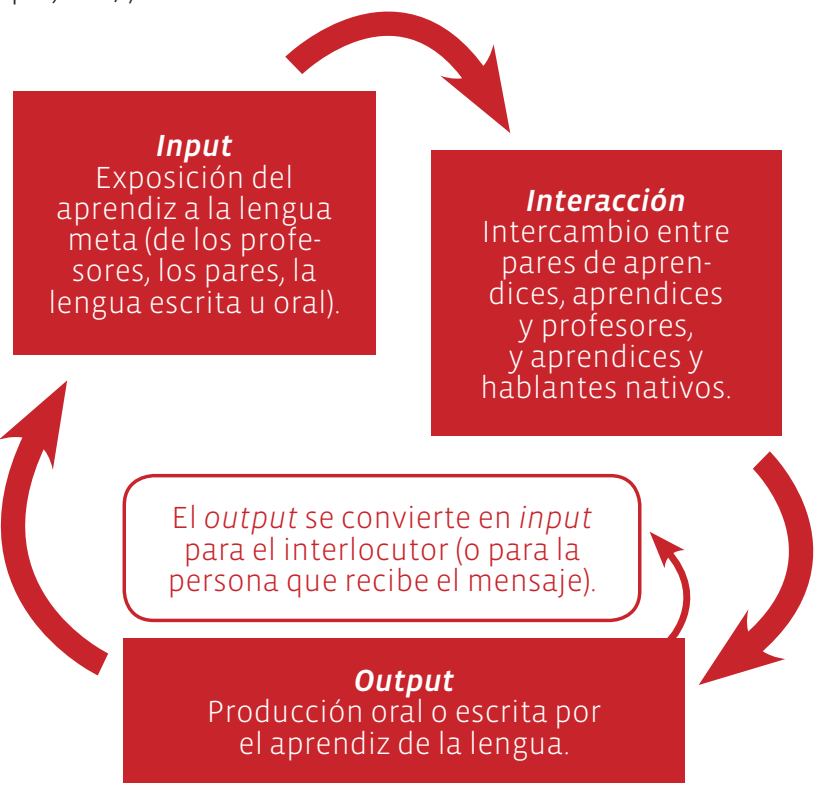


La hipótesis del input comprensible propuesta por Krashen (1985) influyó en el desarroIlo de la hipótesis de la interacción, puesto que el acceso a un input comprensible es necesario para lograr de manera exitosa la adquisición de una segunda lengua (SL). La adquisición es el producto del procesamiento del input y del output (Swain, 1985). El output corresponde a la producción oral o escrita del alumno (Ellis, 2000). La figura 2 representa la relación que se da entre el input, la interacción y el output.

En este contexto, la inclusión de los recursos tecnológicos permite implementar tareas donde el estudiante puede interactuar con otros estudiantes de la lengua objeto de estudio o incluso con hablantes nativos de dicha lengua. La tecnología en este tipo de modalidades posibilita el acercamiento entre el estudiante y la lengua objeto de estudio.

\subsection{Enfoque basado en tareas}

El enfoque por tareas constituye, en palabras de Estaire (2007), un movimiento de evolución natural dentro del enfoque comunicativo. La autora señala que es lógico que un enfoque que comenzó en la década de los 70 evolucione y adquiera nuevas formas en la medida que se continúa reflexionando sobre la lengua. En este enfoque, la tarea es el punto de partida para la programación de una unidad didáctica. Se denomina tareas a "aquellas actividades para las cuales utilizamos la lengua en nuestra vida cotidiana” (Long, 1985). Por su parte, Skehan (1998) define la tarea como una actividad en la que el significado es lo primordial. Las tareas pueden incluir audios, exposiciones orales, lecturas o producción escrita, o la combinación de estas. En este enfoque subyace la idea de que los estudiantes aprenderán mejor una lengua si participan de actividades que conllevan una interacción auténtica (Bachman, 1990). Es decir, si la lengua se utiliza lo más parecido posible a como se usa fuera de la clase. La cuestión es cómo lle- var esto al aula de lengua extranjera. Distintos autores han dividido las tareas de aula en dos clases (Estaire, 2007):

- Tareas de comunicación: Son unidades de trabajo en el aula que se centran en el significado, no en aspectos formales de la lengua. Importa más lo que se expresa que las formas lingüísticas utilizadas para hacerlo.

- Tareas de apoyo lingüístico: Se centran en los aspectos formales de la lengua, en las formas lingüísticas. Actúan como soportes de las tareas de comunicación, capacitando a los alumnos desde el punto de vista lingüístico para la realización de las tareas de comunicación que han sido programadas.

En el caso del Aula Virtual del Instituto Cervantes, el diseño se basa en la metodología comunicativa, específicamente, basada en un enfoque por tareas y en un enfoque centrado en la acción como lo plantea el Marco Común Europeo de Referencia para las Lenguas (MCER, 2002). Por lo tanto, las actividades que se proponen se orientan a trabajar las competencias generales y las competencias comunicativas de la lengua (Juan Lázaro, 2009). De acuerdo con los planteamientos de este enfoque, en cada tema se propone una tarea final, por lo que cada una de las micro-tareas que se propone en las lecciones 0 unidades prepara al estudiante para que realice con éxito la tarea final. En el estudio que presentamos a continuación, el enfoque de lengua también se sustenta en el enfoque por tareas, teniendo en cuenta los postulados de Willis y WiIlis (2007) y de Estaire (2007), considerando, además, las temáticas propias de la cultura chilena.

\section{Metodología de investigación}

Acorde con la problemática planteada en esta investigación, esto es, mejorar las habilidades comunicativas de los estudiantes de español como lengua extranjera (ELE) a través de una aplicación tecnológica en un contexto semipresencial (b-learning), se llevó a cabo el desarrollo, 
la implementación y la evaluación empírica de una aplicación tecnológica para ELE.

\subsection{Desarrollo de la aplicación Tradicio- nes Chilenas}

Los supuestos lingüísticos sobre los cuales se sustenta el desarrollo de la aplicación son el enfoque por tareas y la hipótesis de la interacción. Por consiguiente, los contenidos y actividades reflejan situaciones comunicativas auténticas donde el estudiante puede involucrarse en el uso real y significativo de la lengua. A través de textos interactivos, audios, videos e imágenes dinámicas se proporciona un input comprensible para el nivel en el que se encuentran los alumnos.

El diseño de la aplicación se articula con la definición del perfil del estudiante, los objetivos del aprendizaje y los contenidos lingüísticos. El perfil se delimitó en función de los estudiantes que realizan el intercambio estudiantil con la Universidad de Concepción. Los objetivos de este intercambio son complementar los estudios de pregrado y posgrado en Chile y, a su vez, perfeccionar el conocimiento lingüístico del español como LE. De acuerdo con lo señalado, la aplicación Tradiciones Chilenas se orientó hacia el nivel Bı del español como LE. En consonancia con ello, los objetivos, los contenidos y las lecciones de la aplicación se desarrollaron acorde con los descriptores del nivel B1 especificados en el MCER (2002). En este aspecto, se consideró la comunicación como eje principal, con el fin de promover el mejoramiento de la competencia comunicativa de los estudiantes.

\subsubsection{Contenidos lingüísticos y culturales}

La aplicación Tradiciones Chilenas cuenta con tres lecciones articuladas en nueve secciones. Cada lección gira en torno a un tema principal, los cuales son: 1) la música chilena, 2) la artesanía chilena y 3) la literatura en Chile. La evaluación de esta aplicación se centró en la lección de música chilena (ver figura 3).

\section{FIGURA 3}

Interfaz aplicación Tradiciones Chilenas

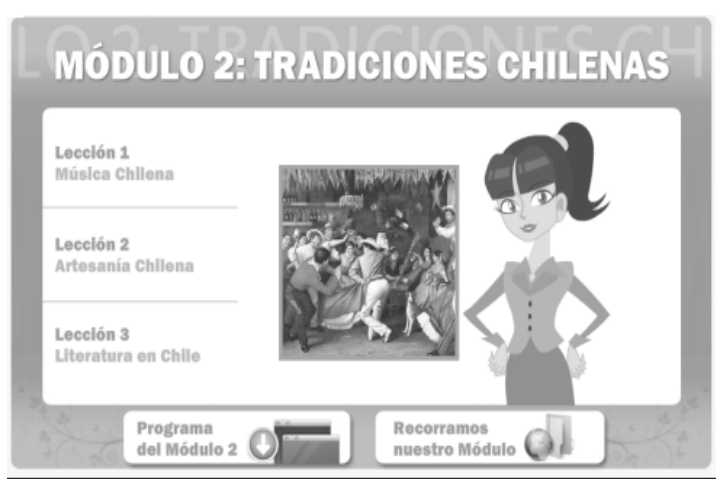

\subsubsection{Criterios de selección de los conte- nidos lingüísticos y tipos de tareas}

Las tareas se desarrollaron de acuerdo con los supuestos teóricos propuestos por Willis y Willis (2007) y Sheila Estaire (2007). Para ello, se diseñaron las pre-tareas, las tareas de apoyo lingüístico y las tareas de comunicación acordes con el Enfoque por tareas como se ilustra y explica a continuación.

Al comienzo de cada lección, se elaboró la sección de cultura como el espacio propicio para diseñar las pre-tareas (ver figura 4). Esto permite una preparación al estudiante en relación con el tema central de la lección. Dichas pre-tareas parten con la observación y escucha de un video o imágenes ilustrativas para luego discutirlas en la clase presencial, en el foro o en el blog.

\section{FIGURA 4}

Pre-tarea para la sección de Cultura

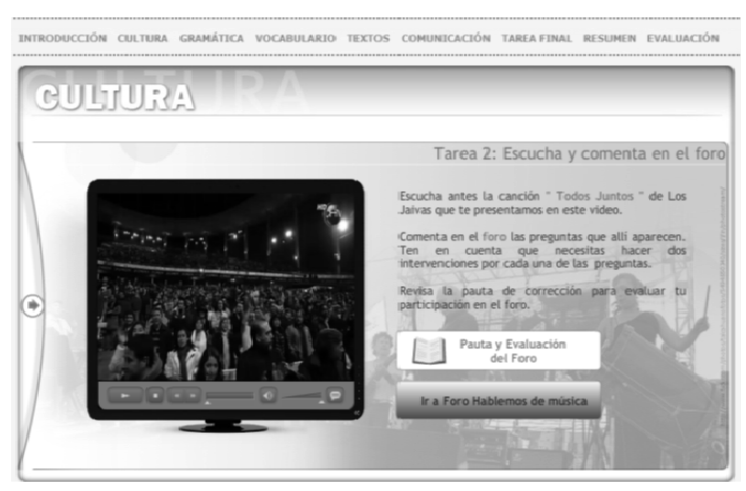


En el caso de la lección de música, se realizó una pre-tarea a partir de un video del grupo musical chileno Los Jaivas. Los estudiantes comparten sus opiniones en la clase presencial y luego escriben sus comentarios en el foro de la lección (ver ejemplo de esta pre-tarea en el anexo 1).

Las tareas de apoyo lingüístico son el soporte de la estructura lingüística de la lengua, por lo que preparan al estudiante en función del uso adecuado de la lengua. Por ejemplo, en la lección de música, la sección de gramática estuvo acompañada de tareas estructuradas a partir de diálogos, y la sección de textos incluyó la escritura de resúmenes a partir de la lectura de textos narrativos y argumentativos (ver anexos 2 y 3 ).

Como puede apreciarse en la figura 5, el diálogo está relacionado con el tema principal de la lección. Además se contextualiza la gramática dentro de una situación comunicativa, que en este caso es una conversación entre amigos. Cada tarea de apoyo fue pensada para promover el uso de la lengua en un contexto comunicativo auténtico.

\section{FIGURA 5}

Tarea de apoyo lingüístico

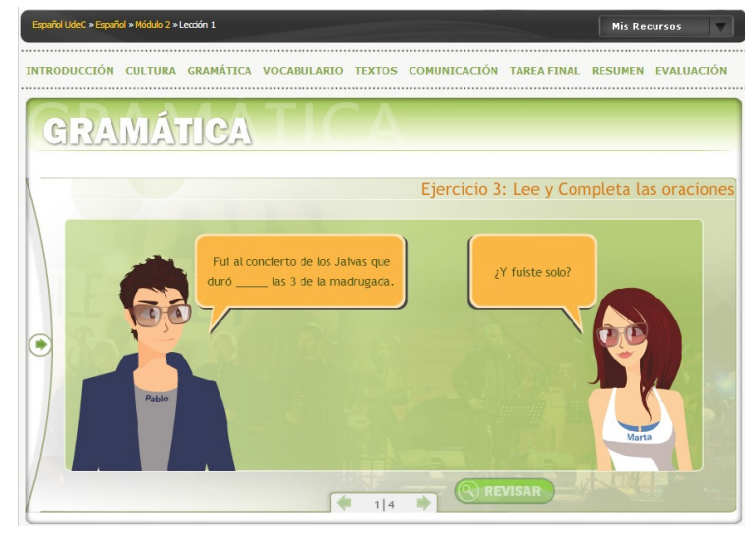

Con respecto a las tareas de comunicación, estas se enfocaron al uso de la producción oral y la comprensión auditiva. Por ejemplo, en la sección de comunicación se diseñaron tareas relacionadas con las formas de expresión oral tales como el comentario, la opinión, el reporte de un acontecimiento por medio de un informe, entre otros.

En la figura 6 se realiza una tarea a partir de la elaboración de un comentario sobre el tema específico de la lección. Esta tarea se contextualiza a partir de la comprensión auditiva, donde se seleccionaron audios insertos dentro de una tradición chilena. En este aspecto, el estudiante no sólo practica la comprensión, sino que además conoce aspectos tanto lingüísticos como culturales. Por lo tanto, la tarea conduce a la inclusión de las características del sistema lingüístico y su contextualización en situaciones culturales de la vida real.

\section{FIGURA 6}

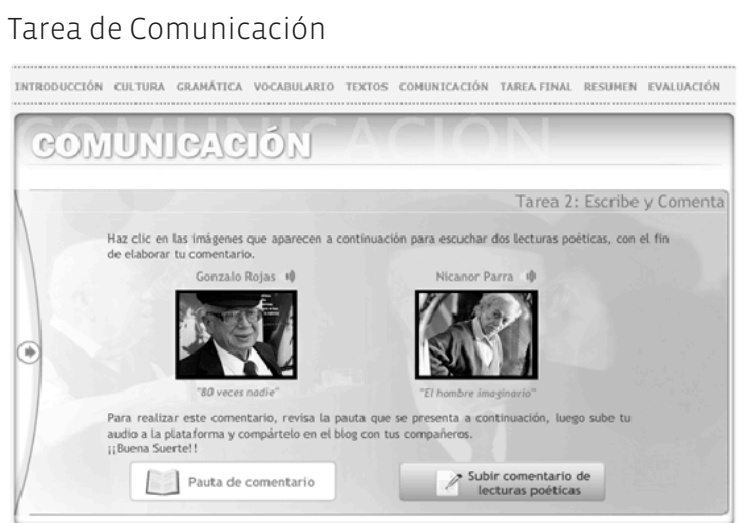

\subsection{Implementación de la aplicación Tradiciones Chilenas}

El desarrollo de la aplicación Tradiciones Chilenas se implementó en la plataforma de interacción Moodle y se alojó en el AULA VIRTUAL ELE-TUTORA (Ferreira, 2010). La aplicación puede utilizarse en tres modalidades de enseñanza: 1 ) "presencial" en un laboratorio computacional de la universidad, 2) "no presencial", para aquellos estudiantes desde otros países y 3) "semipresencial", destinado para el estudio y realización de tareas tanto en el aula de clases como fuera de ella.

La plataforma Moodle proporciona varios recursos que permiten desarrollar actividades de forma dinámica e interactiva. Algunas de ellas 
son, por ejemplo, el envío de tareas en línea, uso del chat para la interacción entre los estudiantes, entre otros. Como puede observarse en la figura 7, las aplicaciones del Aula Virtual están acompañadas de una presentación, en la cual se explican los objetivos, los contenidos lingüísticos y culturales y los logros de aprendizaje que se espera obtener.

\section{FIGURA 7}

Aplicaciones del Aula Virtual

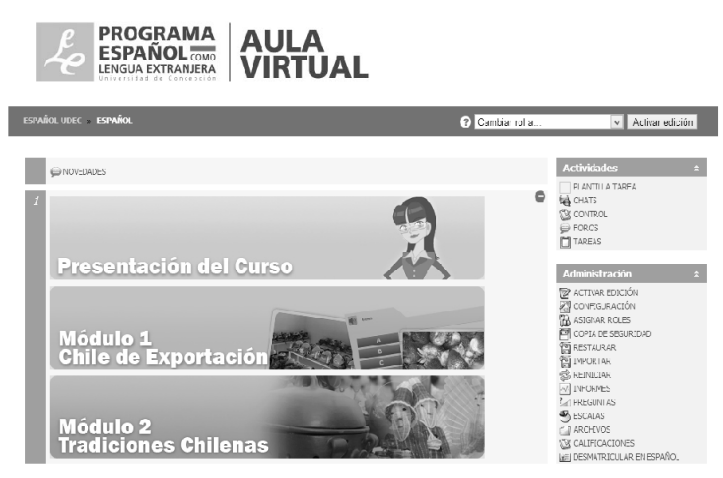

Por otra parte, en el panel derecho, los estudiantes pueden ingresar a su perfil, modificarlo, y activar su blog. Esta primera entrada a la aplicación es simple y amigable, lo que permite una mayor interacción con facilidades de acceso e intercambio de información.

\subsubsection{Los recursos tecnológicos}

La plataforma Moodle cuenta con diversos recursos, los cuales permiten una mejor interacción entre los estudiantes. En este sentido, para la aplicación Tradiciones Chilenas dichos recursos se constituyen en herramientas valiosas que no sólo permiten la interacción, sino también el intercambio intercultural. Es decir, los estudiantes tienen la oportunidad de expresar sus costumbres, su visión cultural y su conocimiento a través del blog, el chat y el foro. De esta manera, enriquecen tanto su bagaje lingüístico como intercultural.

En la figura 8 se muestran los recursos tecnológicos de los que dispone la aplicación Tra- diciones Chilenas: el foro, el chat, el diccionario, el blog y las noticias. Con cada uno de ellos se diseñaron actividades que facilitaron la interacción y el desarrollo de situaciones comunicativas.

\section{FIGURA 8}

Recursos de la aplicación Tradiciones Chilenas

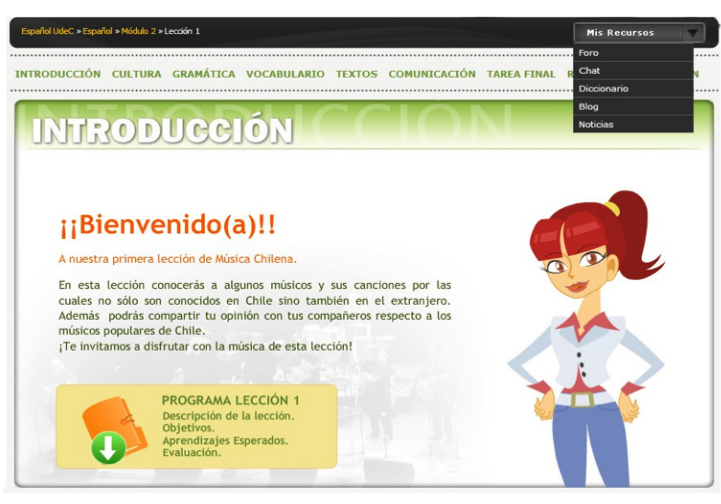

\subsection{Evaluación de la aplicación Tradicio- nes Chilenas}

La evaluación de la aplicación se realizó a través de un estudio empírico con diseño cuasiexperimental y a través de la administración de un cuestionario de satisfacción de usuario.

\subsubsection{Estudio empírico: diseño cuasiex- perimental}

Con el objetivo de determinar la efectividad de las propuestas teóricas y metodológicas de adquisición y enseñanza de segundas lenguas (hipótesis de la interacción, enfoque por tareas y modelo metodológico de (ALL), se llevó a cabo un estudio de tipo cuasiexperimental sin grupo control.

\subsubsection{Pregunta de investigación}

La pregunta de investigación que orienta este estudio es: ¿Se puede mejorar las habilidades comunicativas en ELE en un contexto mediatizado por la tecnología? En relación con esta pregunta se especificó la siguiente hipótesis. 


\subsubsection{Hipótesis}

La operacionalización de un modelo semipresencial basado en la hipótesis de la interacción y enfoque por tareas mejora las habilidades comunicativas en ELE.

\subsubsection{Variables}

Se consideró como variable independiente el uso de tareas mediatizadas por la tecnología en una modalidad semipresencial. Como variable dependiente, el mejoramiento de las habilidades comunicativas en ELE.

\subsubsection{La muestra}

La muestra se constituyó por 21 estudiantes de nivel intermedio del curso ELE nivel BI. Las edades de los alumnos fluctuaban entre los 19 y 28 años (promedio de edades, 22 años). Todos ellos pertenecientes a distintas universidades que tienen convenio con la Universidad de Concepción, como se puede observar en la siguiente tabla:

TABLA 1

Lengua materna de los estudiantes

\begin{tabular}{c|c}
\hline Lengua materna & $\mathbf{N}^{\circ}$ alumnos \\
\hline Alemán & 11 \\
\hline Inglés & 5 \\
\hline Francés & 4 \\
\hline Portugués & 1 \\
\hline
\end{tabular}

\subsubsection{Diseño del experimento}

Teniendo en cuenta el principio del enfoque por tareas, que pone énfasis en que las habilidades y actividades que se tratan deben corresponder a los intereses y dificultades lingüísticas que presentan los estudiantes, se llevó a cabo un análisis de necesidades. De esta manera, se pudo conocer el perfil de los estudiantes respecto a sus intereses temáticos. Se les pidió que escribieran una lista de temas que les gustaría tratar en el curso de español. La mayoría de temas propuestos fue de índole cultural, siendo los más representativos la música y el turismo. Dado que los estudiantes se encontraban de intercambio en la Universidad de Concepción se consideró un mayor número de clases presenciales en la distribución de las sesiones, como se muestra en la tabla 2.

\section{TABLA 2}

Modelo semipresencial para la enseñanza de ELE

\begin{tabular}{c|c} 
Modalidad & $\mathbf{N}^{\circ}$ sesiones \\
Presencial & 5 \\
\hline No presencial & 3 \\
\hline
\end{tabular}

Según lo que se observa en la tabla 2, el proceso de intervención lingüística contempló ocho sesiones; de estas, 5 se realizaron presencialmente en la sala de clases y 3 de forma no presencial mediante la interacción entre el estudiante y el ambiente del aula virtual.

\subsubsection{Proceso de intervención lingüís- tica}

La tabla 3 describe de manera exhaustiva el modelo metodológico utilizado en el proceso de intervención. La primera sesión se realizó de forma presencial con la finalidad de introducir al estudiante en la temática de la lección a trabajar. Se seleccionó la lección de música chilena atendiendo a los intereses de los estudiantes (según análisis de necesidades). En esta primera clase se llevaron a cabo las pre-tareas; la primera fue escuchar una canción y luego comentarla en el foro de la lección (ver ejemplo de esta pre-tarea en anexo 1), y la segunda hacer una búsqueda biográfica de distintos músicos chilenos (en parejas). Ambas actividades estaban vinculadas a lo que el estudiante tendría que realizar después en la tarea final.

En la segunda sesión, no presencial, los alumnos continuaron la tarea de búsqueda ini- 
ciada la clase anterior con el objetivo de preparar el informe (según pauta definida) sobre algún personaje del ámbito musical chileno, el cual debían presentar de forma breve en la tercera clase. Como se observa esta fue una tarea de soporte para la tarea final, ya que los alumnos tenían que exponer e interactuar en relación con el músico que habían elegido. Las clases 4 y 5 se centraron en la habilidad escrita. Cada alumno tenía que leer un texto acorde con una temática musical y luego escribir un resumen (ver ejemplos de resúmenes escritos por los sujetos en anexos 2 y 3 ). Previo a esto, se les proporcionó la estructura de este tipo de texto y algunas formas gramaticales que necesitarían para elaborar de mejor manera el resumen.

En la clase 6, no presencial, los estudiantes tuvieron que leer un texto (disponible en el blog del Aula Virtual) de carácter informativo sobre los diferentes tipos de música según la zona geográfica de Chile. El objetivo de esta tarea es que a partir del texto leído los alumnos puedan indagar más sobre la música y bailes desarrollados en alguna zona específica de Chile y elaborar una presentación oral sobre esto. Las clases 7 y 8, presenciales, corresponden a la tarea final de este proceso de intervención lingüística. En estas sesiones cada estudiante presentó la zona que había seleccionado y los argumentos de su elección frente a sus compañeros (ver muestra de una tarea final en anexo 4). En este tipo de actividad se busca que el alumno sea capaz de integrar lo aprendido e interactúe con sus interlocutores (compañeros y profesora), lo cual se verá reflejado en el desarrollo de sus habilidades comunicativas en español.

\section{TABLA 3}

Descripción del modelo metodológico

\begin{tabular}{|c|c|c|}
\hline No. sesión & Modalidad & Descripción de las tareas \\
\hline$-0-$ & Presencial & -Pre-test \\
\hline 1 (2 hrs.) & Presencial & $\begin{array}{l}\text {-Introducción a la lección de música chilena (Aula Virtual). } \\
\text {-Pre-tarea: Revisión de la canción “Todos juntos” de Los Jaivas. Luego, } \\
\text { comentario respecto a la canción y al grupo en el foro de la lección en } \\
\text { interacción con los compañeros y la profesora (ver anexo 1). } \\
\text {-Inicio de búsqueda bibliográfica con el objetivo de preparar un informe } \\
\text { sobre algún músico o banda chilena de interés del estudiante (actividad } \\
\text { en pareja). Esta actividad posibilitaría el desarrollo de la tarea final. }\end{array}$ \\
\hline 2 (2 hrs.) & No presencial & $\begin{array}{l}\text {-Preparación del informe (evaluación formativa) por parte de los } \\
\text { estudiantes a partir de la búsqueda bibliográfica sobre algún músico o } \\
\text { banda chilena. }\end{array}$ \\
\hline 3 (3 hrs.) & Presencial & $\begin{array}{l}\text {-Breve presentación oral del músico chileno seleccionado para elaborar } \\
\text { el informe. Luego de cada exposición se realiza un breve comentario en } \\
\text { el que participan los alumnos y la profesora. }\end{array}$ \\
\hline 4 (2 hrs.) & Presencial & $\begin{array}{l}\text {-Lectura de un texto narrativo sobre un músico chileno a elección } \\
\text { (Víctor Jara, Violeta Parra, Los Prisioneros) para luego escribir un } \\
\text { resumen en el computador (formato txt; ver anexo 2). } \\
\text {-Se entregó una cápsula instruccional con la estructura del resumen. }\end{array}$ \\
\hline
\end{tabular}




\begin{tabular}{|c|c|c|}
\hline 5 (2 hrs.) & No presencial & $\begin{array}{l}\text {-Lectura de un texto argumentativo sobre música: proyecto de ley } \\
\text { que resguarda la música folclórica chilena en la emisión radial. Luego, } \\
\text { escriben un resumen del texto leído en el computador (formato txt), } \\
\text { considerando la estructura del resumen revisada en la sesión anterior } \\
\text { (ver anexo 3). }\end{array}$ \\
\hline 6 (3 hrs.) & No presencial & $\begin{array}{l}\text {-Lectura del texto "Música tradicional de Chile de acuerdo a las distintas } \\
\text { zonas geográficas". Disponible en el blog del Aula Virtual. } \\
\text {-Tarea final: Preparación de una presentación en power point sobre } \\
\text { alguna zona de Chile que cada alumno debía elegir. El objetivo era } \\
\text { que los estudiantes hicieran una búsqueda más exhaustiva de la } \\
\text { música (instrumentos, bailes, etc.) desarrollada en la zona elegida y la } \\
\text { compararan con su cultura de origen. }\end{array}$ \\
\hline
\end{tabular}

\section{7-8 (2 hrs.) Presencial \\ 3.3.1.7. Instrumentos de recolección de datos}

Tarea final: Exposición oral. Cada estudiante presenta la zona sobre la cual hizo la búsqueda, dando a conocer los motivos por los cuales eligió ese lugar. Además, muestra ejemplos de la música, instrumentos y bailes de esa zona y los contrasta con su cultura de origen e interactúa con sus compañeros y profesora al respecto (ver anexo 4).

-o- Presencial Post-test: Prueba al finalizar el proceso de intervención.

Para evaluar el proceso de intervención lingüística descrito anteriormente, se utilizó un pre-test y un post-test, como se describe a continuación:

- Pre-test: Se evaluó las habilidades lingüísticas a través de una prueba que midió las distintas habilidades de los estudiantes en español, esto es, comprensión auditiva y lectora, y producción oral y escrita. La producción oral se evaluó a través de una entrevista. En la comprensión auditiva los alumnos escucharon 4 audios con tres preguntas cada uno, en la comprensión lectora leyeron 4 textos y respondieron también 3 preguntas por cada uno. Las habilidades productivas se midieron a través de una entrevista (oral) y de la escritura de un texto de 15 líneas sobre un tema específico (recomendar algún lugar para visitar).

- Post-test: Una vez finalizado el proceso de intervención, los estudiantes fueron evaluados con una prueba de características similares al pre-test con el objetivo de verificar si hubo algún incremento en cuanto a sus habilidades comunicativas en español. Respondieron la misma cantidad de ítems del instrumento anterior. Las habilidades productivas fueron evaluadas mediante una entrevista y la escritura de un texto de 15 líneas, en el que debían recomendar un músico chileno a un amigo.

\subsubsection{Resultados del estudio}

De acuerdo con la información que se presenta en la tabla 4, a cada habilidad se le asignó un $25 \%$ respecto del total del porcentaje. Dicha tabla muestra los resultados obtenidos por los estudiantes antes de iniciar el proceso de intervención lingüística.

La media obtenida por el grupo, antes de aplicar el proceso de intervención, fue de un $70 \%$ de logro. No obstante, entre los sujetos se presentan algunas diferencias en cuanto a sus resultados, por ejemplo, el sujeto 3 tiene un promedio de $53 \%$, mientras el sujeto 7 tiene un $82 \%$. 
En términos generales, la habilidad mejor lograda por este grupo de estudiantes es la de producción oral con un $82 \%$ de logro. Por el contrario, la menos favorecida es la de comprensión lectora con solo un $46 \%$.

La tabla 5 presenta los resultados que obtuvieron los estudiantes una vez finalizado el proceso de intervención lingüística. Al contrastar los resultados del pre-test y del post-test se observa que la habilidad más favorecida, una vez concluido el proceso de intervención, fue la comprensión lectora con un aumento de un 18\%. La comprensión auditiva aumentó un 14\% y la producción oral incrementó en un $12 \%$ respecto al promedio obtenido por el grupo en el pre-test. De acuerdo con estos valores, es posible señalar que el aumento en el aprendizaje de los estudiantes se debió a la implementación de la me-

\section{TABLA 4}

Resultados del pre-test

\begin{tabular}{|c|c|c|c|c|c|}
\hline No. SUJETO & C. AUDITIVA \% & C. LECTORA \% & P. ESCRITA \% & P. ORAL \% & TOTAL \\
\hline 1 & 75 & 55 & 85 & 70 & 71 \\
\hline 2 & 75 & 55 & 90 & 90 & 78 \\
\hline 3 & 42 & 18 & 75 & 75 & 53 \\
\hline 4 & 67 & 64 & 90 & 90 & 78 \\
\hline 5 & 67 & 45 & 90 & 85 & 72 \\
\hline 6 & 67 & 55 & 70 & 60 & 63 \\
\hline 7 & 83 & 64 & 90 & 90 & 82 \\
\hline 8 & 58 & 27 & 85 & 80 & 63 \\
\hline 9 & 92 & 55 & 80 & 85 & 78 \\
\hline 10 & 83 & 64 & 90 & 85 & 81 \\
\hline 11 & 83 & 55 & 90 & 90 & 80 \\
\hline 12 & 100 & 45 & 70 & 80 & 74 \\
\hline 13 & 92 & 55 & 80 & 90 & 79 \\
\hline 14 & 83 & 55 & 85 & 85 & 77 \\
\hline 15 & 75 & 36 & 70 & 90 & 68 \\
\hline 16 & 83 & 9 & 75 & 80 & 62 \\
\hline 17 & 67 & 36 & 80 & 85 & 67 \\
\hline 18 & 58 & 45 & 60 & 85 & 62 \\
\hline 19 & 33 & 36 & 80 & 70 & 55 \\
\hline 20 & 75 & 45 & 60 & 80 & 65 \\
\hline 21 & 75 & 55 & 90 & 80 & 75 \\
\hline TOTAL & 73 & 46 & 80 & 82 & 70 \\
\hline
\end{tabular}


todología de enseñanza semipresencial basada en la hipótesis de la interacción y el enfoque por tareas. Esto dado que los recursos tecnológicos del Aula Virtual propician la interacción entre los estudiantes y el acceso a mayor información y textos de los que se dispone en la clase presencial, lo cual favorece el desarrollo de la comprensión y producción oral. En este sentido, el progreso considerable de la comprensión lectora se debió al tipo de tareas que los estudiantes desarrollaron durante la intervención, ya que en ellas tuvieron que revisar y leer diversos textos en distintas páginas web para poder llevar a cabo las actividades solicitadas. Una situación similar ocurrió en la comprensión auditiva, ya que los alumnos fueron expuestos a una gran variedad de input auditivo, tanto en la pre-tarea como en las tareas de comunicación.

\section{TABLA 5}

Resultados del post-test

\begin{tabular}{|c|c|c|c|c|c|}
\hline No. SUJETO & C. AUDITIVA \% & C. LECTORA \% & P. ESCRITA \% & P. ORAL $\%$ & TOTAL \\
\hline 1 & 75 & 50 & 85 & 90 & 75 \\
\hline 2 & 83 & 75 & 90 & 100 & 87 \\
\hline 3 & 67 & 50 & 75 & 95 & 72 \\
\hline 4 & 83 & 83 & 90 & 100 & 89 \\
\hline 5 & 92 & 50 & 85 & 90 & 79 \\
\hline 6 & 83 & 58 & 85 & 90 & 79 \\
\hline 7 & 92 & 83 & 90 & 95 & 90 \\
\hline 8 & 92 & 33 & 80 & 90 & 74 \\
\hline 9 & 92 & 75 & 80 & 95 & 86 \\
\hline 10 & 100 & 75 & 90 & 95 & 90 \\
\hline 11 & 83 & 75 & 90 & 95 & 86 \\
\hline 12 & 83 & 42 & 80 & 90 & 74 \\
\hline 13 & 92 & 75 & 85 & 95 & 87 \\
\hline 14 & 92 & 58 & 85 & 95 & 83 \\
\hline 15 & 83 & 75 & 70 & 95 & 81 \\
\hline 16 & 100 & 67 & 75 & 95 & 84 \\
\hline 17 & 83 & 67 & 80 & 95 & 81 \\
\hline 18 & 100 & 50 & 75 & 90 & 79 \\
\hline 19 & 92 & 58 & 85 & 90 & 81 \\
\hline 20 & 92 & 67 & 85 & 100 & 86 \\
\hline 21 & 75 & 83 & 90 & 95 & 86 \\
\hline TOTAL & 87 & 64 & 83 & 94 & 82 \\
\hline
\end{tabular}


Teniendo en cuenta que los estudiantes se encontraban de intercambio en la universidad, se aprovechó las instancias presenciales para que pudieran practicar el español. Es por ello que sustentado en los planteamientos de la hipótesis de la interacción se propuso una serie de tareas comunicativas que propiciaran el intercambio de ideas referidas a músicos chilenos y de su cultura origen, tipos de música de acuerdo a las zonas geográficas de un país, etc., lo cual se refleja en el $12 \%$ de aumento logrado por el grupo. En consecuencia, es posible señalar que la operacionalización de un modelo de enseñanza semipresencial basado en los enfoques por tareas e hipótesis de la interacción es efectivo, por tanto, contribuye a mejorar la práctica de las habilidades comunicativas de los estudiantes.

\subsubsection{Validez estadística}

Con la finalidad de conocer si el aumento logrado por los estudiantes al finalizar el proceso de intervención era significativo estadísticamente, se aplicó la prueba t student para analizar los datos. Esta prueba indica si la diferencia al comparar dos medias de un grupo, en dos momentos, es significativa. Los resultados obtenidos al contrastar el desempeño del grupo en el pre-test y post-test arrojaron un valor de $t=5,2$ con un valor $p=0,0000003$ (3 E-06). Dado el valor significativo que esta prueba arroja, se confirma la hipótesis planteada, ya que hubo un incremento en el desarrollo de las habilidades comunicativas de los estudiantes, luego de haber trabajado bajo la metodología de enseñanza semipresencial.

No obstante, este hallazgo muestra sólo una tendencia, que debería ser corroborada en futuras investigaciones. Esto debido principalmente al número limitado de estudiantes que constituyen la muestra. Esto se explica debido a la problemática del acceso a estudiantes de ELE en nuestro país, en lo particular, esta situación se ha visto afectada en los últimos años debido a los movimientos estudiantiles.
A pesar de ello, la relevancia de perseverar en estudiar esta temática en ELE en nuestro ámbito chileno sigue siendo importante, especialmente cuando los resultados obtenidos muestran que la operacionalización del modelo teórico de CALL en la modalidad semipresencial es beneficiosa para los profesores e investigadores de ELE que se interesan en incorporar y/o probar metodologías de enseñanza que incorporan la tecnología en una modalidad combinada. La adaptabilidad de esta modalidad de enseñanza al ritmo de aprendizaje del estudiante es una de sus mayores ventajas.

\subsubsection{Cuestionario de satisfacción de usuario}

Atendiendo al segundo tipo de evaluación considerada en CALL, esto es, el análisis valorativo del software y las tareas implementadas, se diseñó un cuestionario de satisfacción de usuario con el objetivo de conocer la impresión de los estudiantes, luego de haber trabajado con la aplicación. Este instrumento se elaboró con una escala tipo Likert de cinco puntos, donde 5 corresponde al concepto de valoración "muy bueno", y 1, "muy malo". Las preguntas se orientaron a evaluar las tareas diseñadas y el entorno donde se alojaban los materiales.

En términos generales, según lo que se observa en la tabla 6, los alumnos evaluaron positivamente, tanto el entorno en el cual se encontraban los materiales (Aula Virtual) como también la metodología de enseñanza utilizada. De acuerdo con esto, los aspectos mejor calificados, con puntuación máxima (5 puntos), fueron las actividades de conversación, conocer y hablar con otros estudiantes extranjeros y las tareas desarrolladas en las clases no presenciales. Si bien todos los ítems tienen una evaluación sobre 4 (bueno), es necesario destacar la valoración que hicieron de los tipos de tareas implementadas en el Aula, esto es, comunicativas, de gramática y léxico, textuales, etc., al asignarle una puntuación promedio de 4,7. Asimismo, las tareas de escritura y presentaciones orales que tuvieron que preparar fueron muy 


\section{TABLA 6}

Resultados evaluación valorativa

\begin{tabular}{|c|c|}
\hline INDICADORES & RESPUESTAS \\
\hline $\begin{array}{l}\text { 1. En general, ¿las clases te } \\
\text { parecieron interesantes? }\end{array}$ & 4,2 \\
\hline $\begin{array}{l}\text { 2. ¿Cómo evalúas los tipos de } \\
\text { tareas (comunicación, gramática, } \\
\text { léxico, etc.) implementadas en el } \\
\text { Aula Virtual? }\end{array}$ & 4,7 \\
\hline $\begin{array}{l}\text { 3. ¿Cómo evalúas las instancias } \\
\text { de conversación? }\end{array}$ & 5,0 \\
\hline $\begin{array}{l}\text { 4. ¿Cómo evalúas las tareas de } \\
\text { presentaciones orales? }\end{array}$ & 4,7 \\
\hline $\begin{array}{l}\text { 5. ¿Te gustó conocer y hablar con } \\
\text { otros extranjeros? }\end{array}$ & 5,0 \\
\hline $\begin{array}{l}\text { 6. ¿Te pareció interesante la } \\
\text { revisión de aspectos culturales } \\
\text { (música chilena) en el Aula } \\
\text { Virtual? }\end{array}$ & 4,6 \\
\hline $\begin{array}{l}\text { 7. ¿Cómo evalúas las tareas de } \\
\text { escritura? }\end{array}$ & 4,7 \\
\hline $\begin{array}{l}\text { 8. ¿Te resultó interesante } \\
\text { realizar tareas fuera de la } \\
\text { clase presencial (clases no } \\
\text { presenciales)? }\end{array}$ & 5,0 \\
\hline $\begin{array}{l}\text { 9. En general, ¿cómo te pareció } \\
\text { la metodología semipresencial } \\
\text { (clases presenciales y no } \\
\text { presenciales) utilizada por los } \\
\text { profesores? }\end{array}$ & 4,3 \\
\hline
\end{tabular}

bien calificadas por ellos, con 4,7 puntos. Las preguntas generales respecto al interés por las clases y la metodología semipresencial tuvieron una puntuación más baja respecto a los otros ítems, pero de todos modos se mantiene sobre los 4 puntos promedio, lo cual es un resultado bastante positivo para nuestro estudio.

\section{Conclusiones}

En este trabajo se planteó operacionalizar el modelo metodológico de CALL en cuanto al desarrollo, implementación y evaluación de una aplicación para la enseñanza de ELE. Este es un modelo que considera distintos elementos y componentes que son necesarios a la hora de diseñar una aplicación para la enseñanza de la lengua a través del computador. El desarrollo, implementación y evaluación permiten visualizar de manera detallada la consistencia entre los principios teóricos y metodológicos que orientan el diseño de una aplicación para ELE y sus respectivas actividades.

De acuerdo con la metodología de CALL, la evaluación tuvo como finalidad, primero, observar empíricamente la ejecución de la aplicación por parte de los estudiantes. Segundo, conocer la valoración de ellos respecto a dicha aplicación. En ambos casos, los resultados obtenidos son positivos, ya que, por un lado, evidencian la efectividad del modelo metodológico utilizado y, por otro, una impresión favorable de parte de los estudiantes hacia el ambiente de aprendizaje y las tareas realizadas. Dichos resultados son semejantes a los logrados por Bañados (2006) en la inclusión del modelo b-learning para la enseñanza del inglés como LE, a los obtenidos en los cursos semipresenciales del aula de Cervantes (Juan Lázaro, 2009) y a los del estudio sobre el aprendizaje en inglés en un modelo b-learning, recientemente publicado, de Ferreira y otros (2014).

En términos generales, los resultados muestran que el desarrollo de las habilidades comunicativas de los estudiantes de nivel BI se vio favorecido al utilizar tareas mediatizadas por la tecnología en el contexto de una modalidad semipresencial. Siendo las habilidades de comprensión lectora, auditiva y producción oral las que presentaron un mayor incremento, $18 \%$, $14 \%$ y $12 \%$, respectivamente. Dichos resultados se corroboran además con las impresiones de los estudiantes, quienes valoran de manera positiva las tareas, el entorno virtual y el modelo metodológico utilizado. Sin embargo, como el periodo de tratamiento fue relativamente bre- 
ve (8 sesiones) y la cantidad de sujetos también pequeña, debido principalmente al acceso de muestras de estudiantes de ELE, se sugiere Ilevar a cabo estudios que repliquen esta metodología en el ámbito de ELE para, de esta forma, robustecer la tendencia y evidencia empírica hallada en este estudio.

Finalmente, los resultados empíricos de este estudio, aunque limitados, han sido validados estadísticamente, permitiendo apoyar empíricamente lo planteado a nivel teórico por Hubbard (2004), en lo que compete al incremento en el proceso de enseñanza-aprendizaje de los estudiantes que aprenden en contextos mediatizados por la tecnología.

\section{Referencias Bibliográficas}

BaChman, Lyle, 1990: Fundamental considerations in language testing, Oxford: Oxford University Press.

BAÑADOs, Emerita, 2006: "A blended-learning pedagogical model for teaching and learning EFL successfully through an online interactive multimedia environment", Calico Journal 23(3), 533-550.

Consejo de Europa, 2002: Marco común europeo de referencia para las lenguas, Madrid: Anaya.

Сото, Víctor, 2014: "El Aula Virtual del Español: modelo de «buenas prácticas» para la enseñanza de segundas lenguas a través de Internet", redELE. Revista Electrónica de Didáctica del Español como Lengua Extranjera, 26.

De Basterrechea, Juan y Olga Juan LÁzaro, 2010:“La integración de las Tic en la actividad docente: el aula virtual de español", Monográficos marCOELE 10, 21-32.

EluIs, Rod, 2000: "Task-based Research and Language Pedagogy", Language Teaching Research 4, 3, 193-220.
Egbert, Joy, 2005: "Conducting research on CALL" en Joy Egbert y Gina Petrie (eds.): CALL research perspectives, Mahwah, NJ: Lawrence Erlbaum Associates, 3-8.

Estalre, Sheila, 2007: "La enseñanza de lenguas mediante tareas: principios y planificación de unidades didácticas" [http://www.nebrija.es/ espanolparainmigrantes/flash/ensenar/PDF/ articulo-tareas.pdf, fecha de consulta: 21 de noviembre de 2013].

Ferreira, Anita, Jaime García y Sandra Morales, 2014 : "Using a Task-Based Approach for Supporting a Blended Learning Model for English as a Foreign Language", International Journal of ComputerAssisted Language Learning and Teaching (IJCALLT) 4, 1, 44-62.

Ferreira, Anita, 2010: ELE-TUTORA: Un Aula Virtual para la Enseñanza del Español como Lengua Extranjera. Concepción, Chile: Universidad de Concepción. Ferreira, Anita, 2007: "Bases teóricas para un modelo B-learning de Enseñanza de Lenguas Extranjeras" en Jaime Sánchez (ed.): Nuevas Ideas en Informática Educativa, Santiago de Chile: LOM Ediciones, 3, 272-296.

Ferreira, Anita, 2005: "Modelo Metodológico de una aplicación multimedial en el contexto de la interdisciplina CALL" en Jaime Sánchez (ed.): Nuevas ideas en Informática Educativa, Santiago de Chile: LOM Ediciones, 2, 49-57.

Gass, Susan, Alison Mackey y Teresa PICA, 1998: "The Role of Input and Interaction in Second Language Acquisition”, Modern Language Journal 82, 299-307.

Gass, Susan, Jennifer Behney y Luke Plonsky, 2013 [1994]: Second Language Acquisition: An introductory Course, New York: Routledge.

Gruba, Paul y Don Hinkelman, 2012: Blended technologies in second language classrooms, New York: Palgrave. 
Hubbard, Philip, 1996: "Elements of CALL Methodology: Development, Evaluation, and Implementation" en Martha Penningnton (ed.): The Power of CALL, Houston: Athelstan, 15-32.

Hubbard, Philip, 2004: "Learner Training for Effective Use of CALL" en Sandra Fotos y Charles BrowNE, (eds.): New Perspectives on CALL for Second Language Classrooms, Mahwah, NJ: Lawrence Erlbaum, 45-67.

JaUREGI, Kristi, 2011: "La negociación de procesos de escritura a través de la videocomunicación”, Quaderns de Filologia. Estudis lingüístics 16, 81-103.

Jauregl, Kristi, Silvia Canto y Carmen Ros, 2006: "La interculturalidad a través de la videoconferencia” en Actas del XVI Congreso Internacional ASELE: La competencia pragmática y la enseñanza del español como lengua extranjera, 749-760.

Jauregl, Kristi, José Gómez y Silvia Canto, 2010: “Interacción virtual a través de la videocomunicación y mundos virtuales: dos estudios piloto", MarCOELE, Revista de didáctica ELE 11.

Krashen, Stephen, 1985: The Input Hypothesis: Issues and Implications, New York: Longman.

Lamy, Marie-Noëlle y Regine Hampel, 2007: Online Communication in Language Learning and Teaching, England: Palgrave Macmillan.

LÁzaro, Olga Juan, 2009: "Web 2.0, Comunicación y material didáctico digital para el aprendizaje del Español: El Aula Virtual de Español del Instituto Cervantes y su actualización", RLA. Revista de Lingüística Teórica y Aplicada 47, 2, 13-34.

Levy, Mike y Glenn StockwelL, 2006: CALL dimensions: options and issues in computer assisted language learning, Mahwah, NJ: Lawrence Erlbaum Associates.
Long, Michael, 1985: "A Role for Instruction in Second Language Acquisition: Task- based Language Training" en Kenneth Hyltenstarm y Manfred Pienemann (eds.): Modelling and Assessing Second Language Acquisition, London: Multilingual Matters.

Long, Michael, 1991: "Focus on Form: A design feature in language teaching methodology" en Kees De Bot, Ralph Ginsberg y Claire Kramsch (eds.): Foreign language research in cross-cultural perspective, London: Benjamins.

López, Mercedes y Michéle Ballez, 2014: "La telecolaboración entre grupos heterogéneos en la clase de lengua extranjera", Revista d'innovació educativa, 8-14.

SHIH, Ru-Chu, 2010: "Blended learning using video-based blogs: Public speaking for English as a second language students", Australasian Journal of Educational Technology 26, 6, 883897.

Skehan, Peter, 1998: "Task-based Instruction", Annual Review of Applied Linguistics 18, 268286.

Swain, Merrill, 1985: "Communicative Competence: Some Roles of Comprehensible Input and Comprehensible Output in its Development" en Susan Gass y Carolyn Madden (eds.): Input in Second Language Acquisition, Rowley, MA: Newbury House, 235-253.

Vine, Ana y Anita Ferrelra, 2012: "Mejoramiento de la competencia comunicativa en español como lengua extranjera a través de la videocomunicación”, RLA. Revista de Lingüística Teórica y Aplicada 50, 1,139-160.

WiLlis, Dave y Jane WiLlis, 2007: Doing task-based teaching, Oxford: University Press. 


\section{Anexos}

Con el fin de ilustrar el trabajo desarrollado por los sujetos en estudio, se adjuntan anexos de diversas tareas del proceso de intervención lingüística.

\section{Anexo 1}

Ejemplo de una pre-tarea realizada en la primera sesión del proceso de intervención lingüística. En esta imagen se visualiza la participación de dos estudiantes en el foro del Aula Virtual, denominado "Hablemos de la música chilena", donde los alumnos expresan su opinión sobre la música chilena.

Re: Hablemos de la música chilena

de - lunes, 1 de octubre de 2012, 11:17

La música chilena se toca con muchos instrumentos distintos. Es tambien posible hacer música chilena solamente con un instrumento, con la guitara, por ejemplo. Un cantante chileno que me gusta y solamente canta y toca la guitara es (olvidó su nombre) ... Nuñez (apellido).

A mi me gustan mucha las canciones para que se puede bailar la cueca tambien. Y por supuesto mirar gente bailer la cueca o intentar yo mismo. ;)

Creo que ya oí este canción de Los Jaivas antes y me gustó pero ne supe de quien es pero me gustó mucho. Entonces, después saber de quien es, voy a intentar recibir billetes para un concierto para ver y esucharlos en directo.

Mostrar mensaje anterior | Editar | Partir | Borrar | Responder

Re: Hablemos de la música chilena

de $\quad$ - lunes, 1 de octubre de 2012, 11:24

Creo que en Chile se escucha mucha música y principalmente musica tradicionale. Tambien los giovenes escuchan a menudo la musica de la idioma origen, por ejemplo tambien en la manera de reggae tone más moderna. La música chilena tiene mucho ritmo y temperamento transmitido de los cantantes y de muchos instrumentos que se tocan para la mejor mezcla. A los chilenos les gusta mucho la musica por que la escuchan casi siempre y conozcan muchas canciones y la letra. Por eso cantan frecuentemente y en todos lugares.

Hay tambien aqui mucha musica buena en vivo en la calle y en muchos conciertos. La canción que escuchamos de los Javias no conocía antes. Pero a mi me gusta mucho la cancion. Un grupo armonico, un ritmo bueno y la letra de la cancion es muy global y importante para todo.

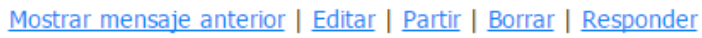

\section{Anexo 2}

Este ejemplo de resumen del sujeto 3 corresponde a una tarea de la cuarta sesión del proceso de intervención. En esta tarea de apoyo lingüístico, los estudiantes tenían que leer un texto narrativo sobre un músico chileno que ellos elegían (Violeta Parra, Víctor Jara y Los Prisioneros) y a partir de esta lectura escribir un resumen en el computador en formato txt.

\section{La humanidad de la Violeta Parra}

Folclorista, cantante, artista y verdadera icono en Chile - el texto es una biografía de Violeta Parra, contando su historia por orden cronológico desde su nacimiento en 1917 hasta el suicidio en 1967. Siempre conectado con los movimientos sociales de Europa o Chile que influyeron su vida, sus obras y su éxito, la biografía trata de relacionar la artista con la época en la que vivió.

Desde sus primeros años, se enfrentó al mundo musical: Sus padres eran profesorde música y campesina guitarrera, Violetta Parra empezó a tocar la guitarra y a escribir sus primeros versos muy temprano, viviendo y trabajando en el campo cerca de Chillán. Ese universo se refleja en sus canciones.

En los 30 se fue a Santiago con la idea de estudiar, pero le interesaba más cantaren los bares y 
pequeñas salas de barrios. Desde 1937 hasta 1948 ella estaba casado con Luis Cereceda - de esta unión nacieron dos hijos, con su segundo pareja, el tapicero Luis Arce, tenía dos hijas.

Violeta Parra en ess época recorrió Chile para haceruna compilación de canciones folcloricas de su país. Hace mucho timepo parte de una red de artistas ocurrió que en 1953 la Radio Chilena la reconoció. En 1954, después de ganar un Caupolicán, fue a Europa donde vivió en Francis.

En 1958 regresó a Chile, inspirada, donde comenzó a pintar, se enamoró de Gilbert Favre y grabó nuevos discos que aumentaron su grado de familiaridad.

Los movimientos sociales y estudiantiles en Europa y Latinoamerica de los 60 influyeron la vida de la Violeta Parra: La gente joven se identificaron con su músics, ella viajó más por Europs, desarollando su arte en varias disciplinas, y reslizó un proyecto de un centro folclórico en Santisgo.

Antes de su suicido, según el sutor, ella se sentía incomprendida porel público chileno y nuncs podría resolver sus problemss. Su vida siempre era conectado con o determinado por movimientos socisles en su slrededor por lo cusl sus canciónes eran muy personales - de hecho eso muestra que no se puede separar entre la persona privada y la persona pública de la Violeta Parra que quizas al mismo tiempo da un razón por su popularidad ya que se siente que su música „consiste" de pura humanidad.

\section{Anexo 3}

Este ejemplo de resumen del sujeto 8 corresponde a una tarea de escritura de la quinta sesión del proceso de intervención. En esta tarea de apoyo lingüístico, los estudiantes tenían que leer un texto argumentativo sobre el proyecto de ley de música folclórica en las radios chilenas y a partir de esta lectura escribir un resumen en el computador en formato txt.

\section{Sujeto No. 8}

\section{RESUMEN - POLÉMICA FRENTE A PROYECTO DE LEY QUE RESGUARDA LA DIFUSIÓN DE LA MÚSICA CHILENA EN LA EMISIÓN RADIAL NACIONAL}

El texto se ocupa con el tema de la cotización de la música emitida en las emisoras. En noviembre 2007 el Diputado UDI Enrique Estay se esforzó por unporcentaje mínimo de $20 \%$ de música nacional en la radio chilena, incluyendo 5\% música folclórica. También se pronunció a favor de multas para los que no cumplirían las normas.

Hubo una gran polémica frente a este proyecto de ley. El presidente Piñera opone a la ley, pero sigue siendo un tema de discusión en el senado.

Críticos dicen que la realización de este proyecto sería contra la libertad de programación, así como cree la Asociación de Radiodifusores de Chile (ARCHI).

En realidad las emisoras no programan lo que quieren escuchar los radioyentes, pero más bien lo que quieren las grandes compañías multinacionales. Ellos exigen que se escucha sus producciones para mejorar la participación en el negocio con que ganan más de 30 millones de dólares a nivel global

Artistas chilenas están excluidos de este lobby, se produce más en compañías nacionales independientes. En manera las artistas chilenos tienen muchos dificultades de acceder a espacios de difusión, por lo tanto no están en igualdad de condiciones para competir 
en un mercado dominado por industrias globalizadas. Otros países de América del sur también tienen cláusulas de protección cultural, por ejemplo Brazil tiene una quotación de 50\% y Argentina de 30\% - para asegurar la difusión de su identidad. Además una quotación de la música en la radio impulsa la industria de música nacional y regional, como también mostró el mismo desarollo con las canales de televisión.

Aunque muchos críticos de la ley dicen que mejor habría otros medidas estatales para apoyar la música chilena, este proyecto abrirá una ventana de difusión para los artistas nacionales, en especial para los emergentes. Por eso músicos como Los Prisioneros también votan en favor de la ley.

Una duda justificada de ARCHI y otros críticos es que si uno pienso en forma consecuente también se necesitaría instaurar quotaciones para otras formas de acceso a la música como para el Internet, los canales de música y la tele. Al fin la fiscalización sea impracticable también.

En resumen el proyecto de Enrique Estay pudo en marcha una gran discusión acerca de la conservación de la cultura chilena, de las consecuencias de la globalización y de un mercado libre en Chile - cuyo resultado sigue siendo muy vago.

\section{Anexo 4}

Ejemplo de una de las diapositivas de una presentación oral del sujeto No. 11 en la tarea final del experimento. Estas presentaciones se realizaron en las clases 7 y 8 . Los estudiantes tenían que elegir una zona de Chile que les hubiese gustado, a partir de la lectura del texto "Música tradicional de Chile de acuerdo a las distintas zonas geográficas", con el objeto de dar a conocer qué zona les agradó y argumentar por qué, como se observa en esta diapositiva.

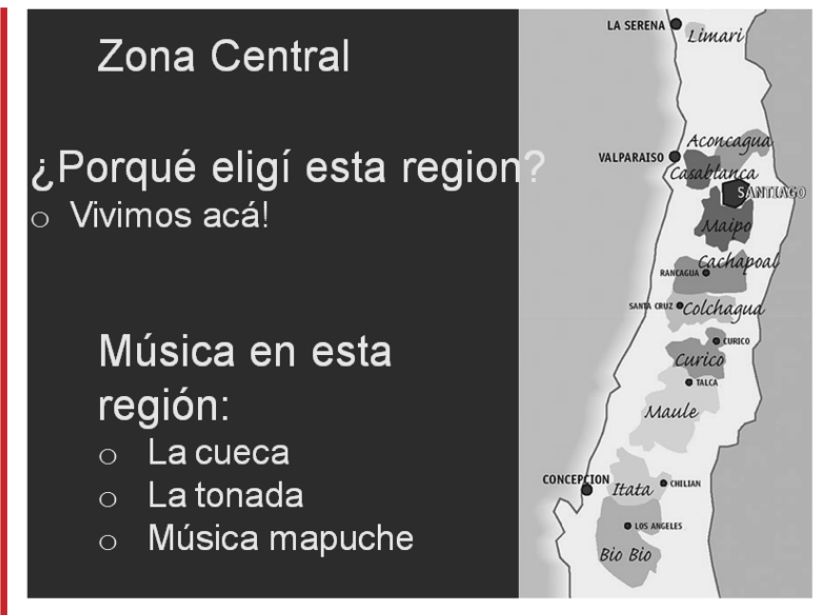

